\title{
Giardiasis: analysis of histological changes in biopsy specimens of 80 patients
}

\author{
G Oberhuber, M Stolte
}

\begin{abstract}
Histological changes in duodenal biopsy specimens from 80 patients infected with Giardia lamblia were defined and compared with changes in duodenal biopsy specimens from 80 randomly chosen "healthy" patients. Villous architecture, the number of intraepithelial lymphocytes, the occurrence of lymphoid follicles and the density of colonisation by $G$ lamblia trophozoites were examined. Grade 1 villous flattening was observed in $41 \%$ of cases positive for Giardia and in $37.5 \%$ of the control cases. The mean content of intraepithelial lymphocytes was 24.5 compared with $22 \cdot 6$ intraepithelial lymphocytes/100 epithelial cells in the positive and negative groups, respectively. Lymphoid follicles were more frequently observed in Giardia positive specimens, but their incidence was not significantly higher compared with that of controls. Twenty five per cent of patients positive for Giardia showed light, $41 \%$ intermediate, and $34 \%$ heavy colonisation by Giardia trophozoites. In $76 \%$ the parasites were observed in all specimens obtained.
\end{abstract}

It is concluded that histological changes induced by $G$ lamblia are not specific, and that two duodenal biopsy specimens might suffice to detect the trophozoites.

Giardiasis is defined as symptomatic or asymptomatic infection by the flagellated parasite Giardia lamblia, which was discovered by Anton van Leeuwenhoek in 1682. Giardiasis is the most common protozoal infection of the intestine and occurs worldwide, from the Tropics to the Arctic.' Despite this, the pathogenetic potential of this parasite has often been questioned. This is due to the considerable differences in the symptoms and histological changes in duodenal biopsy specimens which occur among patients infected with $G$ lamblia. Giardiasis may be totally asymptomatic, but patients may also have diarrhoea which may be associated with intestinal malabsorption. ${ }^{2}$ Clinical, haematological, and biochemical findings do not contribute to the diagnosis of acute giardiasis, ${ }^{3}$ which depends essentially on the identification of the parasite. Giardia infection is therefore confirmed by the demonstration of trophozoites in faecal and duodenal specimens.
Several histological changes associated with giardiasis have been described. ${ }^{4-6}$ Owing to the considerable variability from person to person, however, the small number of cases examined was a shortcoming of these reports. In this study we defined histological changes in duodenal biopsy specimens from 80 patients infected with $G$ lamblia. Villous architecture, the number of intraepithelial lymphocytes, and the occurrence of lymphoid follicles were examined and compared with changes in duodenal biopsy specimens from 80 "healthy" patients. The density of colonisation in several biopsy specimens from each patient was estimated with the aim of establishing the distribution of the parasite in the duodenum.

\section{Methods}

Eighty consecutive cases of giardiasis diagnosed between 1986 and 1988 were examined. The biopsy specimens were submitted for histological evaluation by five different gastroenterologists who obtained a mean of $3 \cdot 1$ (range one to seven) duodenal biopsy specimens. Eighty consecutive cases, histologically classified as "normal", were also analysed. The patients had upper gastrointestinal complaints, and duodenal biopsy specimens were routinely obtained. A mean of $2 \cdot 8$ duodenal biopsy specimens was obtained and examined. To evaluate the number of intraepithelial lymphocytes 40 controls were chosen at random.

Forty three of the patients with giardiasis were female, and thirty seven male. The mean age of the female patients was $47 \cdot 7$ years, that of the male patients 48.9 years. The overall mean age was $48 \cdot 3$ years (range $20-82$ years). Forty five of the controls were female and 29 male. The mean age of the female patients was 52.5 years, that of the male patients $41 \cdot 8$. The overall mean age was $47 \cdot 8$ (range $18-83$ years).

The specimens were fixed in $4 \%$ buffered formalin and embedded in paraffin wax. At least seven $5 \mu \mathrm{m}$ thick sections were stained with haematoxylin and eosin and examined by two different pathologists. In doubtful cases the sections were stained with Giemsa stain. The relative density of colonisation was determined semiquantitatively (range,-+ , $++,+++)$.

To quantify intraepithelial lymphocytes counts of intraepithelial nuclei were made in areas of the slide in which the tissue was well oriented; the plane of the section passed vertically through the epithelium and the basement membrane was readily identified. 
The epithelium was examined at a magnification of $\times 400$. When a section contained a part of a nucleus, this was included in the count if it could unequivocally be classified as epithelial cell or lymphocyte. Intraepithelial lymphocytes traversing the basement membrane were not included. The results were expressed as the number of intraepithelial lymphocytes for each 100 epithelial cells. For each group the median count was calculated. At least 500 epithelial cells were counted.

The degree of villous flattening was assessed semiquantitatively. Grade 0 represented normal villi, grade IV was defined as flat mucosa: grade I was defined as mild villous flattening, which was considered to be a normal variant; the degree of villous flattening in duodenal biopsy specimens with short blunted villi was classified as grade II; and grade III represented a subtotal villous flattening.

Lymphoid follicles were defined as an aggregation of lymphocytes with or without a germinal centre.

The number of intraepithelial lymphocytes in the positive and negative groups were statistically compared using the Wilcoxon rank sum and the Kruskal Wallis tests. The incidence of lymphoid follicles in the two groups was compared using the $\chi^{2}$ test.

\section{Results}

Twenty (25\%) patients showed light colonisation with $G$ lamblia, $33(41 \cdot 2 \%)$ intermediate, and $27(33.7 \%)$ heavy colonisation. In 61 $(76.2 \%)$ cases the parasites were observed in all specimens obtained.

Grade I villous flattening was seen in 33 $(41.2 \%)$ patients with giardiasis. Higher grades of villous flattening were not observed. Grade I villous flattening was also observed in 15 $(37.5 \%)$ of the control cases.

The lamina propria content of lymphocytes and granulocytes was considered to be within the "normal" range in all cases with giardiasis, and in all the controls.

Lymphoid follicles were observed in 31 cases $(38.7 \%)$ with giardiasis and in 16 controls $(21.6 \%)$; the difference was not significant.

The mean content of intraepithelial lymphocytes (table) in cases for positive Giardia was 24.5 (range 10-66) and 22.6 in controls (range 8.8-61.8); the difference was not significant.

Intraepithelial lymphocyte content in Giardia positive and negative duodenal biopsy specimens

\begin{tabular}{lll}
\hline $\begin{array}{l}\text { Number of intraepithelial } \\
\text { lymphocytes/100 epithelial } \\
\text { cells }\end{array}$ & $\begin{array}{l}\text { Percentage } \\
\text { with } \\
\text { giardiasis }\end{array}$ & $\begin{array}{l}\text { Percentage } \\
\text { of } \\
\text { controls }\end{array}$ \\
\hline $5-10$ & 0 & 10 \\
$10-15$ & $18 \cdot 3$ & $22 \cdot 5$ \\
$15-20$ & $26 \cdot 8$ & $22 \cdot 5$ \\
$20-25$ & $20 \cdot 7$ & 15 \\
$25-30$ & $6 \cdot 1$ & 10 \\
$30-35$ & $8 \cdot 5$ & $7 \cdot 5$ \\
$35-40$ & $6 \cdot 1$ & $2 \cdot 5$ \\
$40-45$ & $3 \cdot 7$ & $2 \cdot 5$ \\
$45-50$ & $3 \cdot 7$ & $2 \cdot 5$ \\
$50-55$ & $1 \cdot 2$ & 0 \\
$55-60$ & $2 \cdot 4$ & 0 \\
$60-65$ & $1 \cdot 2$ & 5 \\
\hline
\end{tabular}

\section{Discussion}

G lamblia was readily identified by microscopic examination of duodenal biopsy specimens routinely stained with haematoxylin and eosin. In a few cases Giemsa staining provided additional information; this stain allowed the fine structure of the parasite, which looked like a pear cut lengthwise, to be identified, $G$ lamblia is binucleate, has a ventral disc and four pairs of flagella, and measures $5 \mu \mathrm{m}$ by $20-30$ $\mu \mathrm{m} .{ }^{7}$ Most specimens were heavily colonised by the parasites; only in $20 \%$ was the density of Giardia considered to be low.

In our series the protozoons were detected in $76.3 \%$ of all duodenal biopsy specimens obtained from the patients positive for Giardia. This indicates that the parasites are homogeneously distributed in the duodenum. We therefore conclude that two duodenal biopsy specimens might suffice to diagnose the disease in most cases. On the basis of animal experiments, however, it was concluded that the site of primary infection of the immunocompetent host is the mid-jejunum, which is preferentially colonised, and that fewer organisms infest the duodenum, distal jejunum, or ileum. ${ }^{8}$ If this is true of man diagnosis of the disease through microscopical examination of duodenal biopsy specimens may be difficult in the initial period after infestation.

Giardiasis was distributed equally among male and female patients. This contrasts with experimental studies in mice, in which lower levels of infection were observed in female than in male mice. ${ }^{9}$

Humoral immune responses have an active role in the clearance of $G$ lamblia. ${ }^{10}$ Specific secretory antibodies may be cytotoxic or cause parasite agglutination, inhibition of the motility of flagella, or, possibly, blocking of the attachment to the intestinal epithelium. The humoral immune response was not, however, accompanied by any increase in infiltration by mononuclear cells that could be recognised histologically in the lamina propria. The semiquantitatively assessed content of lymphocytes in the lamina propria was comparable with that of control specimens.

Little is known about cellular immune mechanisms in giardiasis. It has been proposed that in symptomatic giardiasis cell mediated immunity may contribute to mucosal damage. ${ }^{3}$ Intraepithelial lymphocytes are possible candidates: indeed, intraepithelial lymphocytes and lamina propria lymphocytes have been reported to exert a direct cytotoxic and an antibody dependent, cellular mediated cytotoxic effect against Giardia. ${ }^{11}$ Increased numbers of intraepithelial lymphocytes have also been found in symptomatic patients. ${ }^{4-6}$

In our study the mean number of intraepithelial lymphocytes was not significantly increased compared with that of healthy controls. The highest count was 62 intraepithelial lymphocytes/100 epithelial cells, which is compatible with other reports. ${ }^{4-6}$ This result, however, was also obtained in two control specimens. Most Giardia positive biopsy specimens also had "normal" intraepithelial lymphocyte counts. We therefore doubt that the increased 
numbers of intraepithelial lymphocytes were the result of parasitic disease. A possible explanation for increased intraepithelial lymphocyte numbers in symptomatic giardiasis might be bacterial superinfection which could provoke immigration of intraepithelial lymphocytes. In an experimental heterotopic small bowel isograft rat model the number of intraepithelial lymphocytes increased when bacteria accumulated in the grafts, which, functionally, represented a blind loop (Oberhuber G, et al, unpublished observations). In man jejunal infections with Klebsiella, Candida, or other enteropathogens may contribute to malabsorption in chronic giardiasis. ${ }^{12}$

Mild villous flattening was seen in a few cases with giardiasis, but it was also observed in control specimens, indicating that this is a normal variant. More advanced stages of villous flattening were not observed in our series.

In a large proportion of the cases lymphoid follicles were identified in the biopsy specimens. Although lymphoid follicles were more frequently associated with giardiasis, their incidence did not differ significantly in both groups. The follicles were hyperplastic in several of the Giardia positive cases. This finding is difficult to interpret. On the other hand, infestation with $G$ lamblia was frequently found in patients with hypogammaglobulinaemia. ${ }^{13}$ In this case lymphonodular hyperplasia is a morphological feature of immune deficiency. ${ }^{14}$ On the other hand, hyperplastic lymphoid follicles may be a consequence of the immune response to $G$ lamblia. Cells containing IgM are a major plasma cell subtype in lymphonodular hyperplasia. ${ }^{14}$ This is of particular interest as an acute serum IgM immune response in giardiasis has been observed. ${ }^{10}$

In conclusion, giardiasis is not associated with specific histological changes. Despite this, the disease may be diagnosed easily by identifying the infectious agent in duodenal biopsy specimens.

1 Petersen H. Giardiasis (lambliasis). Scand J Gastroenterol 1972; (Supplement 14) 7:7-14.

2 Pickering LK, Woodward WE, DuPont HL, Sullivan P. Occurrence of Giardia lamblia in children day care centers. J Pediatrics 1984;104:522-6.

$3 \mathrm{Kraft}$ SC. The intestinal immune response in giardiasis. Gastroenterol 1979;76:877-9.

4 Wright SG, Tomkins AM. Quantitation of the lymphocytic infiltrate in jejunal epithelium in giardiasis. Clin Exp Immunol 1977;29:408-12.

5 Rosekrans PMC, Lindeman J, Meijer CJLM. Quantitative histological and immunohistochemical findings in jejunal biopsy specimens in giardiasis. Virchows Arch (Pathol Anat) 1981;393:145-51.

6 Ferguson A, McClure JP, Townley RRW. Intraepithelial lymphocyte counts in small intestinal biopsies from children with diarrhoea. Acta Pediatr Scand 1076;65: 541-6.

7 Feely DE, Erlandsen SL, Chase DG. Structure of the trophozoite and cyst. In: Erlandsen SL, Meyer EA, eds. trophozoite and cyst. In: Erlandsen SL, Meyer EA, eds. Giardia and giardiasis. Biology, pathogenesis

8 Gillon F, Ferguson A. Changes in the small intestinal mucosa in Giardiasis. In: Erlandsen SL, Meyer EA, eds. Giardia and giardiasis. Biology, pathogenesis and epidemiology. New York: Plenum Press, 1984:163-83.

9 Roberts-Thomas IC, Mitchell GF, Anders RF, et al. Genetic studies in human and murine giardiasis. Gut 1980;21:397-401.

10 Goka AJK, Rolston DDK, Mathan VI, Farthing MJG. Diagnosis of giardiasis by specific IgM antibody enzyme linked immunosorbent assay. Lancet $1986 ; \mathrm{i}: 184-6$.

11 Kanwar SS, Ganguly NK, Walia BNS, Mahajan RC. Direct and antibody dependent cell mediated cytotoxicity against Giardia lamblia by splenic and intestinal lymphoid cells in mice. Gut 1986;27:73-7.

12 Healy GR. The presence and absence of Giardia lamblia in studies on parasite prevalence in the USA. In: Jakubowski W, Hoff JC, eds. Waterborne transmission of giardiasis. Springfield, Illinois: National Technical Information Service, 1979:92-101.

13 Ament ME, Rubin CE. Relation of giardiasis to abnormal intestinal structure and function in gastrointestinal

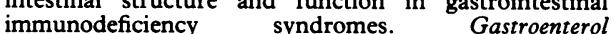
1972;62:216-26.

14 Webster ADB, Kenwright S, Ballard J, et al. Nodular lymphoid hyperplasia of the bowel in primary hypogammaglobulinaemia: study of in vivo and in vitro lymphocyte function. Gut 1977;18:364-72.

15 Nagura H, Kohler PF, Brown WR. Immunohistochemical characterisation of the lymphocytes in nodular lymphoid hyperplasia of the bowel. Lab Invest 1979;40:66-73. 